There are some important points to this letter, however, particularly regarding what is referred to as the non-medicalisation of bereavement.

We are ignoring our duty as professional carers if we do not concern ourselves with the imminently bereaved and are just brushing under the carpet something we find difficult to deal with by withdrawing from our responsibility to bereaved loved ones. We invite relatives, without coercion or callousness, into the resuscitation room and give information in a compassionate way. Infringement of autonomy occurs when a relative or patient is deprived of the information he or she needs for autonomous decision making. It is unacceptable medical paternalism to withhold that information in the fear that it will cause the patient or relative harm.

1 Mitchell MH, Lynch MB. Should relatives be allowed in the resuscitation room? J Accid Emerg Med 1997;14:366-9.

2 Adams S, Whitlock M, Higgs $R$, et al. Should relatives be allowed to watch resuscitation? $\mathrm{BMJ}$ 1994;308:1687-92.

3 Hanson C, Strawser D. Family presence during cardiopulmonary resuscitation: Foote Hospital emergency department's 9 year perspective. J Emerg Nurs 1992;18:104-6.

\section{Anterior glenohumeral dislocation}

EDrToR,-I would like to make a few comments on the very informative article by $A$ Gleeson on shoulder dislocation. ${ }^{1}$

I am one of the many people who reduce shoulder dislocation without injections of drugs but I do not choose this method for physician convenience. It is possible to achieve pain free reduction of the dislocation within a few minutes of the patient's arrival in the accident and emergency (A\&E) department without the use of injected drugs. It simply needs explanation, reassurance, a quiet room, use of a simple relaxation or hypnosis technique, and gentle manipulation.

Relaxation reduces muscular spasm and therefore pain. Entonox given before manipulation promotes relaxation.

The fact should be emphasised that most dislocations can be reduced without use of force and that traction is not necessary. The patient should be informed of this as it helps them to relax. It may take 10 minutes of slow movement to achieve reduction but in a relaxed and confident patient this procedure can be pain free.

It also means that an early attempt at reduction can be made before radiography is considered: there are no complications of slow gentle movement. If the patient is clearly in discomfort then the attempt is stopped and the traditional method used.

I would like to point out that $\mathrm{D}$ and $\mathrm{E}$ in fig 2 are in the wrong order.

Internal rotation is not part of the manoeuvre itself: it simply puts the arm into the position where it can be immobilised. If there is no resistance to internal rotation across the chest then reduction has been achieved. If there is resistance and pain then the dislocation persists.

Can I suggest that the gold standard for shoulder dislocation is reduction:

- Without undue pain

- Without injections of potent drugs

- As soon as possible after arrival in the A\&E department

- Without further damage because force is not used

- Without the complications of benzodiazepines or opiates necessitating admission.
This is achievable: my own experience is that at least half of shoulder reductions can be done this way once the basic techniques become familiar. The success rate improves with practice, familiarity, and confidence.

I wholeheartedly endorse the view that reduction of shoulder dislocation should be a distress free experience and that force should not be involved but disagree that "gold standard" means use of potent drugs.

ALAN LANNIGAN

Accident and Emergency Department, Crosshouse Hospital, Kilmarnock KA1 OBE

1 Gleeson AP. Anterior glenohumeral dislocation: what to do and how to do it. J Accid Emerg Med $1998 ; 15: 7-12$

\section{The author replies}

I agree with Mr Lannigan that it is indeed possible to achieve a relatively pain free reduction of a dislocation within a few minutes of the patient's arrival in $A \& E$ without the use of injected drugs by encouraging the patient to relax and to avoid sudden painful movements of the arm during manipulation. As a minimum though, I would recommend that all patients should be offered a trial of Entonox which will certainly facilitate the reduction process in the majority of cases. It is probably unwise to attempt reduction of an anterior glenohumeral dislocation without prior radiography as there may be a medicolegal battle over whether a fracture evident on a postreduction film was present before reduction or occurred as a result of the reduction manoeuvre. The only circumstance where I would reduce an anterior dislocation without prior radiography would be in recurrent dislocators who have not fallen onto the arm and who sustained the dislocation with a combination of simple abduction and external rotation.

\section{Do we need to be propped up with protocols?}

EDrToR,-Stedman's Medical Dictionary defines protocols as ..."a precise and detailed plan for the study of a biomedical problem or for a regimen of therapy". ' Clinicians argue for and against protocols but seemingly their number is increasing every day. We wondered how many protocols could be drawn up for an accident and emergency (A\&E) department. Using the index of the Cambridge Textbook of Accident and Emergency Medicine as a list, ${ }^{2}$ we selected protocols based on symptoms, signs, diagnoses, and therepaeutic modalities relevant to British A\&E departments.

We excluded conditions which are rare and not serious, administrative and local protocols, tropical diseases, and specialist conditions, for example Salter-Harris fracture classifications. We did not subdivide every major diagnosis, for example atrial fibrillation was not divided into acute and chronic. We did not consider protocols for the patient without an established diagnosis.

Four hundred and two potential protocols were identified of which 280 were deemed essential for every department.

While protocols may enhance quality of care, save time, make medical practice more focused and scientific and reduce litigation, they may also produce more "cookbook" type medicine, inhibit free thinking, and make deviation from protocols open to litigation. Will delay in initiating treatment due to protocol consultation not jeopardise a patient's critical condition? Will it be possible for a jun- ior doctor, or even a senior one, starting in the A\&E department to study and remember all protocols and practise accordingly? By practising protocol based care in the A\&E department are we not admitting that that training or experience or supervision (or all three) are lacking?

The results of our survey, because of the large numbers of protocols involved, suggest protocol based emergency care will be difficult. It is therefore even more important that training is improved and supervision readily available in $\mathrm{A} \& \mathrm{E}$ departments.

F KHAN

D HUGHES

B MCNICHOLL

Accident and Emergency Department, Royal Hospitals, Grosvenor Road,

Belfast BT12 6BA

1 Stedman's medical dictionary. Baltimore: Williams and Wilkinson, 1995: 1446.

2 Skinner D, Swain A, Peyton R, et al. Cambridge textbook of accident and emergency medicine. textbook of accident and emergency medicine. 251-1271.

\section{CS "gas" is not a gas}

EDITOR,-The case report by Breakell and Bodiwala usefully highlights several dangers of CS. ${ }^{1}$ However, by constantly referring to the agent as a gas they reduce the logic of many of their arguments. CS is a solid (melting point $94-95^{\circ} \mathrm{C}$ ), though it may behave as a gas when dispersed as a fine powder from a pyrotechnic device. In Britain, however, CS is deployed dissolved in a solvent. When the solvent has evaporated, solid crystals of CS remain and may be deposited on the skin. This is usually the situation when the patient reaches hospital. Fanning with air, wiping exposed areas with dry tissue, and removal of clothing will be the most effective remedies. Clearly this would not be appropriate or adequate decontamination after exposure to a gas. By using the term CS and not calling it CS gas, people will understand the logic behind the emergency care of exposed victims.

JOHN HENRY

Department of Accident and Emergency Medicine, St Marys Hospital, Praed Street, London W2 $1 \mathrm{NY}$

\section{The authors reply}

We agree entirely with Professor John Henry's comments that CS "gas" is not a gas, and reference to this fact was made in the discussion. ${ }^{1}$ We used the term CS gas due to the popularity and widespread use in the medical literature, not as a description of its chemical properties. We made the point that fanning with air (electric fan) caused contamination of the accident and emergency department and CS powder (crystals) converting to a solution on the surface of the eye should be managed by eye irrigation rather than air currents. ${ }^{2}$

1 Breakell A, Bodiwalwa GG. CS gas exposure in a crowded night club: the consequences for an accident and emergency department. J Accid accident and emergency dep

2 Yih JP. CS gas injury to the eye. BMJ 1995;311: 276 .

\section{Fire}

EDIToR,-On Sunday 5 October 1997 at 3.00 pm we were working in our emergency department at the Derbyshire Royal Infirmary, Derby. The fire alarm sounded and we completely evacuated the department into the car park. We quickly sent one very ill patient to 